\title{
Floristic diversity of swamp meadows affected by the process of murshing
}

\author{
(INDEX: 10-15/2010 Copernican Letters ${ }^{\circledR}$ Vol 1) \\ Katarzyna Kołodziejczyk, Klara Tomaszewska \\ Uniwersytet Przyrodniczy we Wrocławiu \\ katarzyna.kolodziejczyk@up.wroc.pl
}

\begin{abstract}
Research involved 3 swamp meadows affected by murshing process: Czarnocin III and Lubczyńskie Eegi located in Goleniowski district and Pucka Island situated within the borders of Szczecin. There were recognized, in total, 42 species of herbaceous plants representing 7 phytosocjological classes. Particular areas differednot only in the number of species (from 8 on Pucka Island to 24 in Czarnocin III), but also in their percentage composition. The species recognized belong to 17 families. The most numerous one proved to be grasses (Poaceae) - including as many as 13 species On the examined meadows there dominate species from Molinio-Arrhenatheretea class. Environmental requirements of these species were quite similar.
\end{abstract}

\section{Introduction and objectives}

Disusing swamp meadows contributes to further degradation of swamp soil which becomes affected by continuously advancing murshing process involving peat as well as swamp vegetation growing there $[1,2,3,4]$. Peatland drying is accompanied by peat - bog subsidence phase, connected, among others, with the release of considerable amounts of nitrogen compounds. The consequences of subsidence process were discussed by many authors - Okruszko [5, 6], Kucharski [7], Soczewka [8], Wołejko [9], Piórkowski and Rychtalski [10], Tomaszewska [11]. The effect of swamp soils dehydration is continuous decrease in peat layer thickness (called nudation) and forming mursh - mineral and mursh soils. They characterize grain or node - like structure, typical for mursh, and more advantageous aerobic conditions which accelerates peat degradation As an effect environmental conditions undergo alteration which, in turn, leads to replacing water - swamp vegetation by the species requiring dryer medium - so called substitute species and communities. In the case of abandoned post - swamp meadows, they are usually species of lower usability value.

The aim of the work was the assessment of floristic diversity of swamp meadows affected by murshing process and determination of the effect disused plant habitats exert on their value in the view of their biodiversity.

\section{Material and methods}

Environmental study was conducted in July 2009. Three objects - previous swamp meadows localized in the region of Szczecin: Czarnocin III, Lubczyńskie Łęgi and Pucka Island were subjected to analyses in Szczecin. In selected objects there was determined thickness of organic layer with the use of a sampling stick and there was prepared floristic records. Each meadow was provided with 3 records involving the area of $8 \mathrm{~m}^{2}$ in the vicinity of peat sampling holes. Plants names were introduced after Mirek et al. [12],while classification to phytosociological classes followed elaboration by Matuszkiewicz [13]. On the basis of ecological numbers by Zarzycki [14] there were determined such parameters as: life form of species (f), as well as environmental requirements regarding light $(\mathrm{L})$, soil moisture $(\mathrm{W})$, 
fertility (trofism-Tr), soil reaction (acidity-R) and the content of organic matter $(\mathrm{H})$.

\section{Results and discussion}

\subsection{Floristic characteristics of species}

Analyzed post-swamp meadows differed in thickness of organic soil (tab. 1),

Tabela 1

Thickness of mursh horizon of anlysed objects

\begin{tabular}{|l|l|}
\hline OBJECTS & $\begin{array}{l}\text { THICKNESS } \\
\text { ORGANIC } \\
\text { [cm] }\end{array}$ \\
\hline Czaronocin III & 50 \\
\hline Łęgi Lubczyńskie & 40 \\
\hline $\begin{array}{l}\text { Wyspa Pucka wa } \\
\text { Szczecinie }\end{array}$ & 30 \\
\hline
\end{tabular}

wchich influenced the composition of herbaceous plants species. There were, in total, recognized 42 species of vascular plants, yet their number varied in particular investigation objects. The poorest, in terms of floristic values, proved to be Pucka Island with its merely 8 species, while the highest diversity of species, numbering 24, featured Czarnocin III (Tab. 2, Fig. 1).

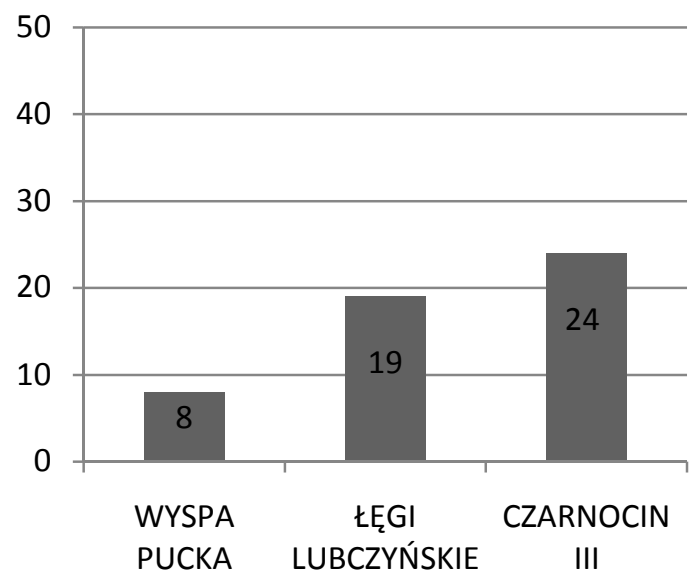

Fig. 1. Number of species of analysed objects

Floristic combination occurring on the examined meadows seems to be interesting. There were found 2 plant species common for 3 analyzed objects, namely: common stinging nettle (Urtica dioica) and Calystegia sepium. The presence of nitrophilous common stinging nettle confirms the increase in nitrogen content originating from the process of peat murshing. Two meadows characterized 5 common species: Phalaris arundinacea, Symphytum officinalis, Galeopsis tetrahit, Juncus effusus and Cirsium arvense. As many as 35 species, providing for $83,3 \%$ of their total number, occurred only once, yet they were found in different sites on the examined areas (Fig. 2.).

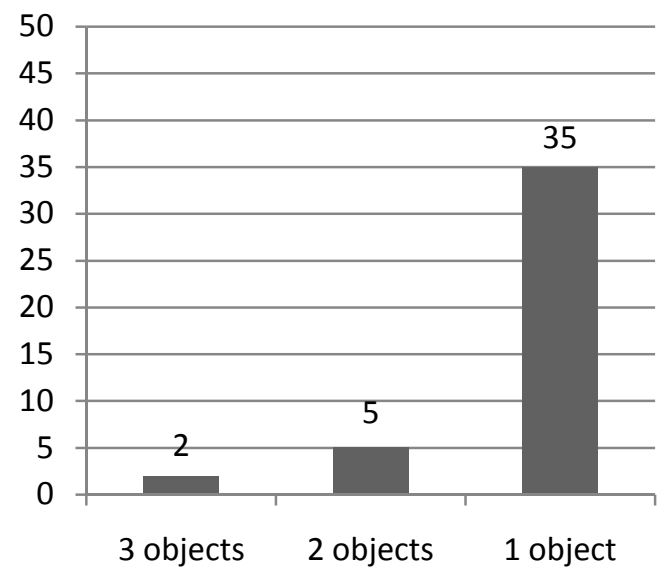

Fig. 2. Recuration of species of analysed objects

Pucka Island was the only place to find tall cat grass (Arrhenatherum elatius) and Alium angulosum. Łęgi Lubczyńskie featured 12 species which did not occur anywhere else, among others, Carex riparia, timothy grass (Alopecurus pratensis), or Lycopus europaeus. In Czarnocin III there were recorded 17 such species. Biodiversity as high as that points to floristic richness of post - swamp meadows and, at the same time, it confirms individual character of particular objects. Similar floristic diversity was recorded for Lower Silesia $[3,4]$.

Plant species found on the meadows subjected to investigation belong to 17 botanical families and 7 phytosociological classes. The most numerous proved to be grasses family (Poaceae) - including as many as 13 species (Fig. 3., Fig. 4.). 


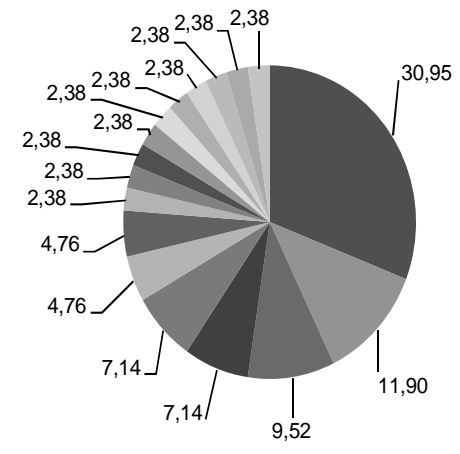

$$
\begin{aligned}
& \text { - POACEAE Trawy } \\
& \text { - ASTERACEAE Złożone } \\
& \text { - } \text { LAMIACEAE Wargowe } \\
& \text { - FABACEAE Motylkowate } \\
& \text { - POLYGONACEAE Rdestowate } \\
& \text { - JUNCACEAE Sitowate } \\
& \text { - APIACEAE Selerowate } \\
& \text { - BORAGINACEAE Szorstkolistne } \\
& \text { - CARYOPHYLLACEAE Goździkowate } \\
& \text { - RUBIACEAE Marzanowate } \\
& \text { - RANUNCULACEAE Jaskrowate } \\
& \text { - CYPERACEAE Turzycowate } \\
& \text { - URTICACEAE Pokrzywowate } \\
& \text { - CONVOLVULACEAE Powojowate } \\
& \text { - LILIACEAE Liliowate } \\
& \text { - PLANTAGINACEAE Babkowate } \\
& \text { - ONAGRACEAE Wiesiołkowate }
\end{aligned}
$$

Fig. 3. The percentage share of families on all investigated areas

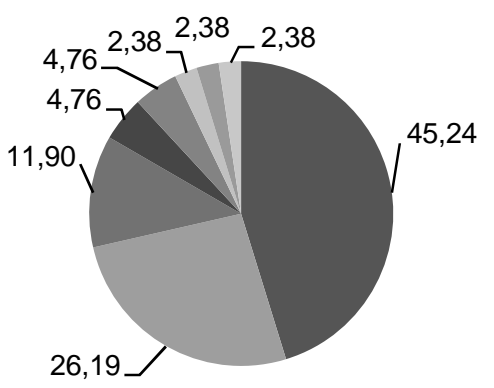

\footnotetext{
- Molinio-Arrhenatheretea

- Artemisietea vulgaris

- Phragmitetea

- Agropyretea intermedio-repentis

- Stellarieta mediae

Bidentetea Tripartiti

- Festuco-Brometea

- Bez przydziału do klasy
}

Fig. 4. The percentage share of of phytosociological classes on all investigated areas

Alterations of water relations of a soil resulted in nearly complete elimination of so - called water - swamp species. On the examined areas there were recorded only 5 species belonging to Phragmitetea R.Tx. et Prsg. 1942 class, including common reed
(Phragmites australis), Carex riparia, reed canary grass (Phalaris arundinacea), marshy hog's fennel (Peucedanum palustre)and Rumex hydrolaatum. Among 42 recognized species as many as $45,2 \%$ represent MolinioArrhenatheretea R.Tx. 1937class, refered to as so - called species of half - natural and anthropogenic sward meadows and pastures communities on mineralized and partly - dried murshes originating from low peat [13] (Fig 4.). Communities of this class were formed as a result of water relations regulations, as well as of management combined with sowing of grass mixtures featuring high pasture quality. On some of the examined areas it is already possible to meet a few representatives of these grass species. On Pucka Island it is Arrhenatherum elatius, Alopecurus pratensis, while Poa pretensis also growth in Łęgi Lubczyńskie, similarly to Dactylis glomerata and Phleum pratense. The phenomenon of replacement the species of high pasture value, requiring higher soil moisture by species adjusted to dryer environment was also reported by Kozłowska [2] in meadow communities situated in river valleys of Central Poland (among others in Grabia, Pilsiacand Dabrówka). On the meadows subjected to investigation there also occur species belonging to such classes as: Atremisietea vulgaris Lohm. and Prsg. et R. Tx. 1950 - nitrophilic communities of large perennials growing in ruderal and water reservoirs borders environment, as well as Bidentetea tripartiti R. Tx., Lohm. et Prsg. 1950 - moderately nitrophilic, mainly natural communities of summer therophytes growing on the borders of inland water reservoirs which dry out in the summer [13]. Increased share of nitrophilic species indirectly indicates advancing peat degradation (murshing) which leads to the increase in nitrogen content in soil. Each of the analyzed post - swamp meadows characterizes different composition of species from particular phytosociological classes (Fig. 4.), confirming their high floristic diversity and stresses unique character of the examined meadows. 


\subsection{Environmental requirement}

Analysis of environmental requirements, based on ecological numbers according to Zarzycki [14], proved that recognized species form a group of nearly identical environmental requirements (Tab. 2), where the following parameters are dominant:

- life form HEMICRYPTOPHYTES (buds on ground level),

- light index $(\mathrm{L})=4$ - moderate light,

- soil moisture index $(\mathrm{W})=3-5$ - fresk, most and wet conditions

- trophism index $=3-4(\mathrm{Tr})$ - refers to soil (water) moderately poor (mesotrophic) and rich (eutrophic),

- soil acidity index $(\mathrm{R})=4-5$ - neutral soils, acid $6<\mathrm{pH}<7$ and basic soils, $\mathrm{pH}>7$

- organic matter content index $(\mathrm{H})=2-3$

- mineral - humous soils and soils rich in organic matter, organogenic soils.

\section{Conclusions}

- Disused agriculturally and degraded swamp soil not always features floristic poverty of plant habitats. There were recognized 42 plant species belonging to 17 botanical families and 7 phytosociological classes.

- Results of our investigation indicate that analyzed meadows are different. Among recognized species only 2 of them: Urtica dioica and Calystegia sepium occurred in all the examined areas.

- Most plant species growing on these meadows characterize the same environmental requirements.

- Appearance of mursh layer brings about the invasion of non - peat - forming plant species which stimulates increased biodiversity.

\section{BIBLIOGRAPHY}

[1] T. Brandyk, T. Gnatowski, R Oleszczuk., J. Szatyłowicz, D. Szejba, „Postęp w badaniach właściwości fizycznych gleb torfowo-murszowych". [w]: Biernacka E. (red.) Torfowiska i mokradła. Wyd. SGGW Warszawa: 91 - 112, 2007.

[2] T. Kozłowska, „Zmiany zbiorowisk łąkowych na tle różnicowania się warunków siedliskowych

w charakterystycznych obszarach dolin rzecznych Polski Centralnej". WodaŚrodowisko-Obszary Wiejskie, Rozprawy naukowe i monografie nr 14 . Wyd. IMUZ, s.208, 2005.

[3] M. Podlaska, „Zbiorowiska roślinne nieużytkowanych łąk pobagiennych na Dolnym Śląsku, ich przemiany oraz wartość gospodarcza i przyrodnicza". Praca doktorska, manuskrypt, Katedra Botaniki i Ekologii Roślin, Uniwersytet Przyrodniczy we Wrocławiu., ss. 145, 2009.

[4] M. Podlaska, K. Tomaszewska, „Wartość użytkowa i przyrodnicza zbiorowisk roślinnych nieużytkowanych łąk pobagiennych „Parowa” koło Bolesławca”. Ann. Sil. XXXVI: 79 - 90, 2009.

[5] H. Okruszko, "Zjawisko degradacji torfu na tle rozwoju torfowisk". Zesz. Probl. Post. Nauk Roln., 2: 69 - 111, 1956.

[6] H. Okruszko, „Przeobrażanie się mokradeł pod wpływem odwodnienia". Zesz. Probl. Post. Nauk Roln., 372: 251 - 269, 1991.

[7] L. Kucharski, „Roślinność łąk w województwie skierniewickim i jej zmiany w bieżącym stuleciu". Przegl. Przyr. VIII, 1 / 2: 63 - 72, 1997.

[8] B. Soczewka, ,Kierunki przemian zbiorowisk roślinnych na siedliskach zabagnionych i podmokłych w dolinie rzeki Kostrzyń w woj. siedleckim". Przegl. Przyr. IX, 1/2: 223 - 226, 1998.

[9] L. Wołejko, „,Biodiversity changes after abandonment of grassland of peatlands. [w: ] Ilnicki P. (red.) Restoration of carbon sequestrating capacity ond biodiversity in abandonmed grassland of peatland on Poland". Wyd. Akademii Rolniczej w Poznaniu: 58 - 76, 2002.

[10] H. Piórkowski, M. Rychtalski, „Siedlisko glebotwórcze i gleby”. [w:] Renaturyzacja dolin rzecznych na obszarach zmeliorowanych wyłączonych z produkcji 
rolniczej na przykładzie obiektów Rudni, Małynka, Tyniewicze. Wyd. IMUZ: 28-41, 2003.

[11] K. Tomaszewska, „Zmiany w składzie gatunkowym fitocenoz na porzuconych łąkach pobagiennych". Ann. Sil. XXXII: 103116, 2003.

[12] Z. Mirek i inni, ,Flowering plants and pteridophytes of Poland". A checklist. Instytut Botaniki im. W. Szafera, PAN, Kraków, 2002.

[13] W. Matuszkiewicz „Przewodnik do oznaczania zbiorowisk roślinnych Polski”. PWN, Warszawa, s. 537, 2001.

[14] K. Zarzycki , H. Trzcińska-Tacikowa, W. Różański., Z. Szeląg, J. Wołek \& U. Korzeniak, „Ecological indicator values of vascular plants of Poland", Polish Academy of Sciences, Kraków, 2002.

Badania współfinansowane przez Unię Europejską ze środków Europejskiego Funduszu Społecznego w ramach projektu systemowego pn. „Przedsiębiorczy doktorant inwestycja w innowacyjny rozwój regionu" (Program Operacyjny Kapitat Ludzki, Priorytet VIII Regionalne Kadry Gospodarki, Działanie 8.2 Transfer Wiedzy, Poddziatania 8.2.2 Regionalne Strategie Innowacji). 
The number of species identify on analysed meadows, share of families and environmental requirement

Tabela 2 Explanation of abbreviations: CZAR-Czarnocin III, ŁL-Łęgi Lubczyńskie, WP-Pucka Island

\begin{tabular}{|c|c|c|c|c|c|c|c|c|c|c|}
\hline \multirow{2}{*}{ FAMILY } & \multirow{2}{*}{ SPECIES } & \multicolumn{6}{|c|}{ ECOLOGICAL INDICATOR VALUES } & \multirow[b]{2}{*}{ CZAR } & \multirow{2}{*}{$\measuredangle L$} & \multirow{2}{*}{ WP } \\
\hline & & f & $\mathrm{L}$ & W & $\mathrm{Tr}$ & $\mathrm{R}$ & $\mathrm{H}$ & & & \\
\hline \multirow{13}{*}{ POACEAE } & Phragimtes communis & $\mathrm{G}, \mathrm{Hy}$ & $4-5$ & $5-6$ & $4-3$ & 4 & $2-3$ & + & + & \\
\hline & Phalaris arundinacea & $\mathrm{G}, \mathrm{H}$ & 4 & 5 & 4 & $4-5$ & 2 & + & + & \\
\hline & Holcus lanatus & $\mathrm{H}$ & 4 & 4 & $3-4$ & 4 & 2 & + & & \\
\hline & Poa trivialis & $\mathrm{H}$ & 4 & 4 & 4 & 4 & 2 & + & & \\
\hline & Festuca arundinacea & $\mathrm{H}$ & 4 & $3-4$ & 4 & 4 & 2 & + & & \\
\hline & Poa pratensis & $\mathrm{H}$ & 4 & 3 & 4 & 4 & 2 & & + & \\
\hline & Phleum pratense & $\mathrm{H}$ & 4 & $2-3$ & $3-4$ & $4-5$ & 2 & & + & \\
\hline & Bromus inermis & $\mathrm{H}$ & 5 & $2-3$ & 3 & $4-5$ & $1-2$ & & + & \\
\hline & Dactylis glomerata & $\mathrm{H}$ & 4 & 3 & $4-5$ & $4-5$ & 2 & & + & \\
\hline & Calamagrostis epigeios & $\mathrm{G}, \mathrm{H}$ & 4 & 3 & 3 & 3 & 1 & & & + \\
\hline & Arrhenatherum elatius & $\mathrm{H}$ & 4 & 3 & 4 & $4-5$ & 2 & & &.+ \\
\hline & Alopecurus pratensis & $\mathrm{H}$ & 4 & 4 & 4 & 4 & 2 & & & + \\
\hline & Deschampsia caespitosa & $\mathrm{H}$ & $3-5$ & 4 & $3-4$ & $3-4$ & $2-3$ & & & + \\
\hline \multirow{6}{*}{ ASTERACEAE } & Galeopsis tetrahit & $T$ & 4 & $3-4$ & $3-4$ & 4 & 2 & + & + & \\
\hline & Sonchus asper & $\mathrm{T}$ & 5 & 3 & 4 & 4 & 2 & + & & \\
\hline & Eupatorium cannabinum & $\mathrm{H}$ & $3-4$ & 4 & $4-5$ & $4-5$ & 2 & + & & \\
\hline & Cirsium arvense & $\mathrm{G}$ & 5 & $2-3$ & $3-4$ & $3-5$ & 2 & + & & \\
\hline & Matricaria maritima & $\mathrm{H}, \mathrm{T}$ & 5 & 3 & 4 & 4 & 2 & & + & \\
\hline & Artemisia vulgaris & $\mathrm{H}$ & 5 & 3 & 4 & $4-5$ & 2 & & + & \\
\hline \multirow{4}{*}{ LAMIACEAE } & Galeopsis tetrahit & $\mathrm{T}$ & 4 & $3-4$ & $3-4$ & 4 & 2 & & & \\
\hline & Glechoma hederacea & $\mathrm{G}, \mathrm{H}$ & $4-2$ & $3-4$ & 4 & 4 & 2 & + & & \\
\hline & Stachys palustris & $G$ & $5-4$ & $4-5$ & $3-4$ & 4 & 2 & + & . & \\
\hline & Lycopus europaeus & $\mathrm{H}, \mathrm{Hy}$ & 3 & 5 & 4 & 4 & $2-3$ & & + & \\
\hline \multirow{3}{*}{ FABACEAE } & Trifolium repens & $\mathrm{C}, \mathrm{H}$ & 4 & $3-4$ & 4 & 4 & 2 & + & & \\
\hline & Trifolium hybridum & $\mathrm{H}$ & 4 & 4 & 4 & 4 & 3 & + & & \\
\hline & Vicia cracca & $\mathrm{H}$ & 4 & 3 & 4 & $3-4$ & 2 & + & & \\
\hline \multirow{2}{*}{ JUNCACEAE } & Juncus effusus & $\mathrm{H}$ & 4 & $4-5$ & $4-3$ & 4 & 3 & + & + & \\
\hline & Juncus conglomeratus & $\mathrm{H}$ & 4 & $4-5$ & 3 & 4 & 3 & & + & \\
\hline \multirow{2}{*}{ POLYGONACEAE } & Rumex hydrolapathum & $\mathrm{H}, \mathrm{Hy}$ & $3-4$ & $5-6$ & $4-3$ & $4-5$ & 3 & + & + & \\
\hline & Polygonum amphibium & $\mathrm{Hy}, \mathrm{G}$ & 4 & 6 & $4-3$ & 5 & 2 & & + & \\
\hline \multirow{2}{*}{ APIACEAE } & Angelica archangelica & $\mathrm{H}$ & 4 & 4 & 4 & 4 & 2 & + & & \\
\hline & Peucedanum palustre & $\mathrm{H}$ & $4-3$ & 5 & 3 & 3 & 3 & & + & \\
\hline RANUNCULACEAE & Ranunculus repens & $\mathrm{H}$ & $4-5$ & $4-3$ & 4 & $4-5$ & 2 & + & & \\
\hline CYPERACEAE & Carex riparia & $\mathrm{H}, \mathrm{Hy}$ & $4-3$ & 5 & 4 & 4 & 3 & & + & \\
\hline RUBIACEAE & Galium aparine & $\mathrm{T}, \mathrm{H}$ & $5-4$ & $4-3$ & $4-5$ & 4 & 2 & & & + \\
\hline URTICACEAE & Urtica dioica & $\mathrm{H}$ & $2-5$ & $3-4$ & $4-5$ & 4 & 2 & + & + & + \\
\hline CONVOLVULACEAE & Calystegia sepium & $\mathrm{G}, \mathrm{Hi}, \mathrm{li}$ & 3 & 4 & 4 & 5 & 2 & + & + & + \\
\hline LILIACEAE & Alium angulosum & $\mathrm{G}$ & 4 & 4 & 3 & 4 & 2 & & & + \\
\hline PLANTAGINACEAE & Plantago media & $\mathrm{H}$ & 4 & $2-3$ & $4-3$ & $5-4$ & 2 & + & & \\
\hline ONAGRACEAE & Epilobium parviflorum & $\mathrm{H}$ & 4 & $4-5$ & 4 & 4 & 3 & + & & \\
\hline CARYOPHYLLACEAE & Myosoton aquaticum & $\mathrm{G}, \mathrm{H}$ & $4-3$ & $4-5$ & 4 & 4 & 3 & + & & \\
\hline BORAGINACEAE & Symphytum officinalis & $\mathrm{G}, \mathrm{H}$ & 4 & $4-5$ & 4 & 4 & 2 & + & + & \\
\hline
\end{tabular}

